\title{
Experimental and numerical study of stenotic flows
}

\begin{abstract}
Atherosclerosis has been leading cause of deaths in several countries. Recent technical advances have allowed the investigation of stenotic flows and in understanding the implications with increased severity. Such studies shall provide detailed understanding of flow across stenosis and its progression. In the present study, a large artery representing segment of aorta is considered as test specimen. Experimental study is carried out by generating the pulsatile flow through pulsatile duplicator. Flow across normal and various severities of stenosis such as $25 \%, 50 \%$ and $75 \%$ are studied. Numerical simulation using CFD is also carried out in similar normal and stenosed models. A novel concept of using pulse duplicator to generate pulsatile waves and investigate the different stenosed models has been adopted. Results obtained experimentally and numerically are compared and agree well with that of clinical observations. This study demonstrates significant variation of haemodynamic in post-stenotic region with increased stenosis. Increased pulse pressure, phase lag is observed with increased severity. It is also observed that stenosis greater than $75 \%$ is significant as flow complexity is induced with considerable disturbance even in early and latter part of pulse cycle. Such study shall be useful in understanding the flow changes in stenosis and enhance clinical observation.
\end{abstract}

Keyword: Arterial stenosis; CFD; Haemodynamic; Stenotic flow 